\title{
Towards a Conceptual Framework for Persistent Use: A Technical Plan to Achieve Semantic Interoperability within Electronic Health Record Systems
}

\author{
Shellon M. Blackman-Lees, Ph.D. \\ shellon.lees@gmail.com
}

\begin{abstract}
Semantic interoperability within the health care sector requires that patient data be fully available and shared without ambiguity across participating health facilities. Ongoing discussions to achieve interoperability within the health care industry continue to emphasize the need for healthcare facilities to successfully adopt and implement Electronic Health Record (EHR) systems. Reluctance by the healthcare industry to implement these EHRs for the purpose of achieving interoperability has led to the current research problem where it was determined that there is no existing single data standardization structure that can effectively share and interpret patient data within heterogeneous systems.

The current research used the design science research methodology (DSRM) to design and develop a master data standardization and translation (MDST) model which incorporated the Resource Description Framework $(R D F)$ that allowed seamless exchange of healthcare data among multiple facilities. Using RDF, a universal exchange language, allowed for multiple data models and vocabularies to be easily combined and interrelated within a single environment thereby reducing data definition ambiguity.
\end{abstract}

\section{Introduction}

The successful adoption and implementation of EHR systems have been crucial to the health care industry [41]. With the enactment of the Affordable Care Act (2010), the push for a national health information database continued to be a key discussion point at various levels. However, the reluctance to adopt a comprehensive EHR solution was also very prevalent. One of the primary reasons for this reluctance was the inability of the EHRs to interlink and communicate with each other due to the lack of a comprehensive data standard that facilitated the exchange of data using a common data model [4]. The decision to adopt a common data structure within the health facility to promote interoperability had previously been met with reluctance due to financial concerns as well as barriers related to changes to the existing work flow and training of their staff [15]. Further, while health facilities were making substantial efforts toward the adoption and implementation of health information technology solutions, more effort needed to be applied to improve the health information exchange capabilities.

The existence of several independent data standards repositories such as International Classification of Diseases (ICD), Logical Observation Identifiers, Names, and Codes (LOINC), and Systematized Nomenclature of Medicine (SNOWMED), health care facilities cannot successfully achieve interoperability since there was no cohesive standardization format that could act as a single comprehensive standard for data interpretation and translation of medical terminologies and vocabularies [37]. Interoperability of electronic information remained a tremendous challenge especially with over 100 electronic healthcare information standards that currently exist [28]. Each standard served as a standalone structure with its own unique mapping algorithm which could lead to duplication of meaning and interpretation. Due to this challenge of achieving interoperability, there existed a necessity for a common information format where all participants could speak the same language (standards) and interpret similar processes and vocabularies (translation) thus providing the opportunity to achieve seamless exchange of clinical EHR data among health care entities.

Given the rate of acquisitions and mergers that take place, health facilities were reluctant to change their way of operation thus opting to keep their current practices [23]. As a result, the notion of a common information model implementation and use became illusive. Healthcare facilities tended to adopt their own independent data standards repository which presented a hindrance to the overall goal of achieving interoperability [28]. 


\section{Research problem}

There was currently no single source data standardization model to achieve semantic health data interoperability between heterogeneous systems [37], [46]. Clinical information systems currently use different data standardization terminology repositories (HL7, LOINC, SNOMED) for the exchange of health data and information which was a major barrier to EHR interoperability [37].

Data interoperability was a key factor for seamless information exchange among health information systems [23]. Data interoperability was also impossible to accomplish in the current state due to the lack of a relationship between healthcare data and the different health information systems, a growing concern for healthcare practitioners and facilities since it prevented the provision of better patient care [23]. According to the federal regulation mandate of Health Information Technology for Economic and Clinical Health (HITECH, 2009), data-level interoperability was critical to today's practice which included frequent exchange and storage of patient data between healthcare systems to provide optimal patient care and experience.

In 2014, [50] conducted research in the area of semantic interoperability between clinical systems and the practical application of a reference architecture to the exchange of health information. The research showed that there was no single source practical guideline that will allow semantic interoperability based on the availability of data standardization methods, relevant vocabularies, and standards for interpretation. The researchers further noted that while there had been previous research that indicated a similar problem [6], [31], there still existed no model that was currently implemented to support the different vocabularies, data interpretation algorithms, and mapping tools in a single source environment; they were all stand-alone applications that hindered interoperability among heterogeneous systems.

As the need to exchange healthcare data continued to grow, the inability to share and communicate patient data across these systems became impossible due to the varying data standardization models that were adopted by the health systems which could only ensure interoperability within its own operational domain [37].

The significance of data interoperability between health systems was critical to providing efficient patient care that could improve the accuracy of diagnoses, reduction in the number of duplicated tests results, minimize the occurrence of readmission, and prevent medication errors [14]. Despite the progress that was substantially evident with the enactment of
HITECH (2009), still quite a large number of hospitals and healthcare organizations did not electronically exchange clinical data summaries and other patient information. This lack of interoperability, the researchers explained, limited the goal of patient care optimization and coordination across several entities.

One of the major barriers to electronic health information interoperability was the heterogeneity of clinical data sources that operated on the foundation of data standard models that restricted the exchange of data external to its domain [13]. The research problem hindered the integration of multiple systems that could and were willing to share patient information. A suggested solution to resolving this problem was the combined use of standardized information models (single source concept) that incorporated specific domain concept definitions instead of the generic concepts that were currently included in the EHR architectures [13].

The conceptual basis of the problem outlined in this research could therefore be defined as the lack of a single source for data reference and standardization that would allow seamless data exchange - semantic interoperability -- between different healthcare systems within and outside an organization's domain. The information systems theory that best explained the presence of the research problem defined was the organizational information processing theory which identified the following concepts as its foundational basis: "information processing needs, information processing capability, and the fit between the two to obtain optimal performance" ([30], 263).

In 2013, [38] proposed a framework for data standardization of cardiovascular risk stratification at the domain level into the EHR that automated the workflow process of the clinicians. The framework was based on biomedical ontologies derived from the conceptual model of SNOMED and the heart rate turbulence (HRT) domain. It was explained that the combination of the two structures allowed for new concepts such as ventricular tachograms and sinus oscillation for turbulence slope to be generated, which further allowed for better patient service and performance by the clinicians to provide optimal care.

Expanding on the study conducted by [38] the research developed a common information model based on the medical observations, diagnoses, and medications ontologies derived from multiple data standardization models (HL7, SNOMED, LOINC). The framework proposed targeted the workflow of clinicians at the patient registration and encounter domain levels of multiple facilities that used different data standardization models for data translation and standardization. To achieve interoperability through a common data standardization structure within a single 
environment, where multiple independent data models could coexist, the translation mechanism incorporated the use of the Resource Description Framework (RDF). $\mathrm{RDF}$ is a universal healthcare exchange language that allows multiple data models and vocabularies to be easily combined and interrelated within a single environment thus reducing data definition [45]. The outcome of the research model depended on the implementation of the solution at a hospital corporation that integrated the registration and encounter processes to ensure data consolidation occurs within the single environment.

\subsection{Research questions}

The research sought to answer the following questions:

1. What functionality should the translation model provide to capture the collection and translation of patient data?

2. What evidence of semantic interoperability demonstrated the existence of that functionality?

\section{Research goal}

The goal of the research was to design and develop a master data translation model based on RDF. The translation model provided a framework to exchange patient data that had shared meaning with no ambiguity within the health systems. According to the Healthcare Information and Management Systems Society (HIMSS), semantic interoperability involves the use of data models to communicate data in a way that could be interpreted in the same manner by both the sender and receiver. Thus, the RDF based translation model provided a framework which sought to address two main issues that hindered semantic interoperability - a need for a central standards repository and the ability to effectively translate data between various data models and vocabularies to provide a singular interpretation across entities.

As a universal healthcare exchange language, RDF was ideally suited for data translation and had been identified as an acceptable candidate for data exchange by leaders in healthcare and health [45]. The primary strengths of RDF were that it allowed diverse data to coexist, allowed data models and vocabularies to evolve, and facilitated data transformation in a multischema friendly environment [40], [1]. The positive factors of RDF highlighted by these researchers further reinforced the decision to use RDF to develop a robust interoperable solution that provided the capability to freely exchange patient data within the healthcare sector thus allowing healthcare professionals to make better decisions for each patient.

\section{Research impact}

As the body of knowledge was examined, it was determined that various researchers had also explored this conceptual basis of the problem of EHR interoperability - the lack of a comprehensive data standards model to promote interoperability [1], [2], [43], [46], [22], [18], [44], [10]. While many researched this problem from the perspective of varied concentrated areas of interest, the general consensus remained the same; there still remained a deficiency in the way health information could be exchanged within multiple healthcare organizations across states or even locally.

The impact of the research problem defined could be felt across many healthcare entities especially since the implementation of HITECH (2009), a federal regulation that insisted on the need to promote and adopt the exchange of health information data at a national level by ensuring that electronic health record systems were interoperable. A national survey of hospitals conducted between 2008 and 2012 showed a significant increase in the patient data exchange activity while the clinical data exchange with participants outside the hospital had doubled [14].

According to the U.S. Department of Health and Human Services, the enactment of the HITECH Act of 2009 insisted on the need to promote and adopt the exchange of health information data at a national level by incorporating meaningful use of interoperable electronic health record systems. The need to provide complete and optimal care to patients by having complete access to their health records required that patient data was available and could be shared without ambiguity across participating health facilities. The lack of interoperability among healthcare systems had triggered many discussions and attempts towards finding a solution.

The research was relevant and significant to the goal of providing an interoperable solution that would facilitate the exchange of healthcare data thus providing the best care to patients, a factor that is a requirement based on the Affordable Care Act (2010). The EHR/Health Information Exchange (HIE) Interoperability Workgroup - a group consisting of participants from 19 US states, EHR and HIE vendors - was formed to ensure that the existing standards and guidelines for interoperability between HIE applications could be integrated and be compatible from state to state. This group had identified the issues of interoperability based on the lack of standards and integration protocols that would accomplish the cross 
communication of health data exchange across multiple platforms and users. The solution - a common information model for data standardization and translation - would add to the body of knowledge, a framework that could be expanded to incorporate varying data structures seeking to become interoperable.

\section{Review of the literature}

The organization of the literature review proceeded by examining key factors that were necessary for the development of a comprehensive information model to achieve EHR semantic interoperability. An analysis of the articles compiled for the literature review provided a conclusion that the implementation of a viable EHR interoperability solution would involve significant factors of data standardization and translation which would allow for the exploration of: (a) the current healthcare based standards of EHR interoperability [22], [24], [37], [1], [5]; (b) technical infrastructure which focused on the back-end infrastructure [2], [23]; and (c) how existing EHR interoperability solutions were implemented [27], [33], [34].

Semantic interoperability of healthcare data can significantly improve the quality and efficiency of patient care delivery and improve the overall performance of the healthcare systems within the United States [20]. As such, the foundation of the research stresses the importance of achieving semantic interoperability within the healthcare sector.

Based on the review of the literature, these factors served as the foundational benchmark for the research.

\subsection{Data standardization}

The major barrier to EHR interoperability where clinical information systems use different data models and terminology repositories was investigated by [37]. For this reason, the issue of interoperability persisted since data within these systems were stand-alone and therefore not interoperable. The research also indicated that there was no common understanding or descriptive characteristic of the data represented within these information systems which contributed to the barrier to interoperability. Although there had been several proposed solutions (Federal Health Information Model, Study Data Tabulation Model, Domain Analysis Model, Common Data Model) to solve this issue, [37] explained that they were considered to be data dictionaries or abstract data models which could only ensure interoperability within the boundaries of the operational domain. As such, the limitation of these models prohibit the query services, analysis methods, and the data exchange protocols from achieving a broader range of interoperability because they were designed to run within the data model that was specifically defined by a set of core data elements.

Another research by [28] elaborated on the premise that there was a need for standards that would dictate the seamless exchange of clinical EHR data among participating entities. The research looked at the impact of adopting a common data model for the purpose of data collection and exchange. The foundational framework of the study was based on comparative research studies (CER) that required data from clinical information systems. This investigation added much needed information to the body of knowledge (health care cost reduction, improving health policy decisions, and advancement of health research) since CER studies were heavily dependent on clinical data stored within EHRs and they sought to provide answers to patient details such as treatment, intervention, and exposure on outcomes.

In this comparative analysis study, existing models being implemented by organizations associated with clinical research such as the Observational Medical Outcomes Partnership (OMOP), Analysis Data Model (ADaM), Biomedical Research Integrated Domain Group (BRIDG), the Clinical Data Interchange Standards Consortium (CDISC), and the US Food and Drug Administration (FDA) were compared. In addition to comparing the models to determine their strengths (schema and terminology standardization) and weaknesses (unmapped data and information loss) in the analysis for clinical data for the purpose of syntactic and semantic interoperability, the standards of the different models were also compared based on whether they could be extended, could adequately capture patient personal and clinical data, could be understood by clinical researchers and data analysts, had the capability to use standardized vocabularies, and had analytic methods that were well defined.

The results of the study showed that while most of the models adequately captured patient demographic and clinical data (drugs, procedures, observations, providers, benefit plans, patients details), the data models demonstrated a common weakness, that was, access to the translation vocabularies did require improvement. This observation was evident in the presence of standardized vocabularies and data dictionaries in the OMOP model but the evidence showed that these structures would need to be further defined in the BRIDG and ADaM models. However, the issues related to the successful achievement of semantic interoperability, information loss, and data mediation using the data models would require further exploration. 


\subsection{Technological optimization and modification}

Initially, no tool existed that provided a solution for defining semantic alignment of clinical information between different databases [1]. The problem explored in this research sought to provide a solution that would enhance existing alignment techniques by implementing the Resource Description Framework (RDF) schema that would target context-dependent semantic elements allowing for a more expressive alignment within the data structure. Most of the existing database integration tools only addressed the semantic integration segments at a schema level rather than at a domain level in which elements were linked semantically with other elements that belonged to the same source or object within the ontology. The research problem affected the integration technique of current tools that could only map element-to-element (e2e), a 1:1 mapping between single primitive elements within their context [1].

To conduct the research a design and develop methodology was used to build and test a software tool that implemented a view-oriented approach for aligning RDF-based biomedical repositories. The goal of the research was to create a technological framework that would integrate clinical data in order to develop personalized drugs and therapies for cancer patients based on their genetic profile. A view-oriented tool was used to integrate different RDF-based databases that included clinical trials repositories and Digital Imaging and Communications in Medicine (DICOM) images using the Health Data Ontology Trunk (HDOT) as the target schema. The composition of each alignment consisted of a set of entries each containing one RDF-based view from the physical database and another from the HDOT. The graphical view that was constructed with the tool showed the mappings of two RDF paths - one for the patient (BiopsyAfter) $\rightarrow$ undergoes $\rightarrow$ biopsy and biopsy $\rightarrow$ precedes $\rightarrow$ chemotherapy - which existed on different data sources and the other for the patient (BiopsyBefore) $\rightarrow$ precedes $\rightarrow$ Chemotherapy. Compared to the e2e mapping, which currently exists in other sources, [1] explained that their tool had incorporated the semantic layers (RDF sub-graphs) regarding whether the patient's biopsy was performed before or after chemotherapy whereas the e2e based approach failed to sufficiently represent the data at a similar level.

The results of the test conducted in the research showed that while traditional tools were limited to mapping elements within a single domain, the application of RDF-based models resulted in files that were used from different sources that were successfully translated from data stored in the physical databases into the HDOT common format provided.

\subsection{Current EHR solution implementation}

Research by [33] examined the current EHR practices being implemented within the Department of Veteran's Affairs (VA) and Department of Defense (DoD) health systems. The problem explored in this research stated that integrated systems such as clinical decision support (CDS) systems have not been effectively implemented and have failed to apply key strategies and practices in the areas of usability testing, work process redesign and integration, and inconsistent implementation of their EHRs. The current EHR implementation is deficient especially with the anticipation of emerging opportunities with the enactment of the Affordable Care Act (2010). For instance, the current system would not adequately process patient records that were generated from multiple sources such as VA, DoD, or non-VA/DoD providers and patients.

To conduct the research, 31 operational, clinical, and informatics people in leadership positions were invited to participate in the study; 14 agreed to be interviewed. 30 minutes telephone interviews on topics related to EHRs within the VA and DoD were conducted. The data collected was analyzed and the responses were integrated into meaningful patterns that were placed into two specific common themes/categories which described varying areas of EHR innovation. Among the areas of EHR innovations identified - cognitive support (interface, workflow), information synthesis, teamwork/communication, interoperability, data availability, interface usability, customization, managing information and overall vision - [33] emphasized the factor of interoperability as being the highest priority. The responses received from participants indicated that there was a general consensus among many of the leaders who stressed the importance for the VA and DoD information systems to be interoperable.

The conclusions drawn by [33] explained that while they were able to identify consistent themes that were critical factors to the enhancement of the VA's EHR systems, research was still needed to examine the role of organizational and other contextual factors that would be considered in the redesign of the nextgeneration EHR. These factors would enhance the revised care delivery system and business processes that will meet the challenges of the present as well as the next generation interoperability solution. 


\section{Research solution}

As the analysis of the RDF specifications proceeded, the factors considered in the design of the XDataRDF translation model included the RDF definitions, matching based on defined rules, matching based on name constructs, and matching based on common value inference. The translation of the data reused the RDF definitions of the W3C standards which made the manipulation and transformation of data "homogeneous to a common RDF semantic model" ([42], [189]). The ability to create a common translation model was based on the semantic schema of RDF to determine the characteristics of the data vocabularies' domains and ranges of their properties. Using the RDF classes and properties schema, XDataRDF made data inferences, leading to a common interpretation, based on the data vocabulary values stored in the SQL tables.

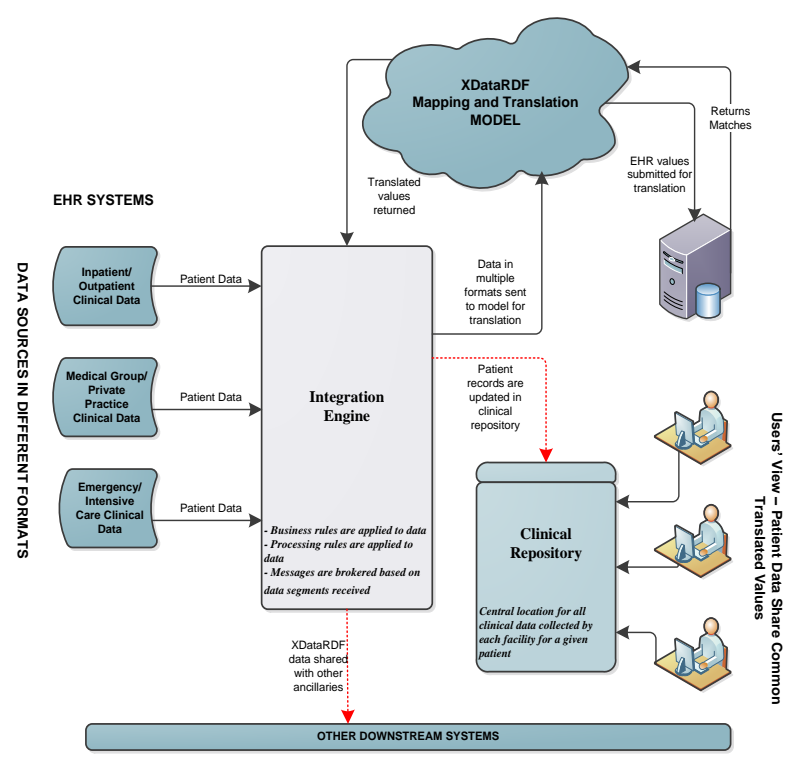

Figure 1. High Level Technical Design of XDataRDF Model

To address the research questions, the high level technical design of the research artifact illustrated in Figure 1 sought to provide the answer. The functional specification of the design captured the flow of data from the EHR systems (data input) to the clinical repository (transformed data output). The process specification of the design demonstrated the flow of data from the EHRs to the integration engine to the mapping and translation model.

\subsection{Technical plan}

The design and development approach maintained through the creation of the XDataRDF translation model adopted the design science research methodology (DSRM), a commonly accepted framework used in design science research [29]. XdataRDF demonstrated the flow of patient data from multiple sources through the EHRs via an integration engine to the target systems. To effectively and thoroughly address the research questions, an organized research approach was taken which expanded on the steps taken to complete the phases of DSRM as outlined in Figure 2.
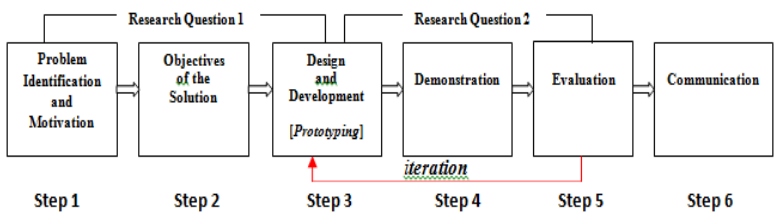

Figure 2. Research Methodology Approach

\section{Step 1: Problem identification and motivation}

After a preliminary research of the topic area and subsequent review of peer-reviewed literature publications and journal articles, online sources, and discussions with focus groups on semantic interoperability within the healthcare environment, two main problems emerged. First, with the enactment of the federal regulation, HITECH (2009), which required the sharing of patient health data among facilities, it was determined that there was no adequate framework to facilitate the standardization and translation of healthcare data to achieve semantic interoperability among the participating facilities ([37], [47], [8]). Second, the goal to achieve semantic interoperability was hindered due to the reluctance of healthcare facilities to modify their existing environments based on factors of cost, changes to operational practices, and adjustments to their training procedures to meet the requirements of this goal ([33], [27]). As a result of these two conflicting issues, the motivation to pursue this research was anticipated and supported.

\section{Step 2: Objective of the solution}

The overall objective of the research solution was to deliver a healthcare data translation model that adequately met the standards and expectations of semantic interoperability of healthcare data. To successfully accomplish this, the design of the solution was based on the problem definition. As such, the 
knowledge of how feasible its functionality would be was a critical factor that was considered in the research [29]. To this end, the research solution sought to accomplish the goal of semantic interoperability incorporating the factors of design, functionality, and usability. These factors which were evident in the model sought to ensure that health care data could seamlessly be exchanged among multiple facilities within a single environment. As such, the steps taken in the research process towards determining the ultimate requirement specifications applied to the design and develop of the research solution were as follows: (A) selection of an expert panel and (B) determination and validation of the requirement specifications to design and develop the research solution.

The selection criteria for the Requirements Panel of experts were based on the following factors knowledge [3], qualification [19], social acclamation [32], and experience [35]. To validate the research solution, the process of the Delphi Method was applied. The Delphi Method is a widely used technique for achieving consensus of opinion from experts within specified fields [21].

To effectively address the research questions, the study executed a two-step process. First, experts were selected from various groups to form two panels Requirements Panel (panel 1) and Development Panel (panel 2). Second, an initial questionnaire which consisted of criteria based on the following categories - design, infrastructure, connectivity -- was distributed to the Requirements Panel anonymously via Google. Using the Delphi Technique, the first research question was addressed by the implementation of the questionnaire iteratively where the research solution requirements were determined and validated. After three rounds of questionnaire distribution, the approved list of criteria was given to the Development Panel.

\section{Step 3: Design and development}

In order to achieve the research goal, the design and development phase expanded on the objectives of the research solution outlined in step 2. As part of the design and development process, a prototype was built using various iterations and increment releases that added to the functionality of the overall solution.

Guided by the Delphi Technique, in the first round of the process, the final reviewed and approved research requirements criteria formulated by Requirements Panel were provided to the Development Panel who conducted the review and analysis of the design and development elements of the research prototype. A preliminary prototype design was developed and presented to the Development Panel via a web link. Each expert reviewed the design to verify that the requirement criteria of the proposed research have been applied.

The second round began with the preliminary prototype design approved by the Development Panel. Round two provided the Development Panel with incremental developed releases of the prototype that satisfied all of the research requirements. For each increment presented, the experts verified the functionality of each component using a predefined test plan. The test plan allowed the Development Panel to determine the accuracy of the data and process flows based on the requirements criteria. Each participant reviewed the prototype and provided feedback based on the functionality and conformance to the requirement specifications. Functional recommendations and feedback submitted by the Development Panel were applied to the development and a subsequent release of the refined prototype was communicated to the panel to be reviewed. Each incremental release evolved to a complete functional artifact with each set of iteration. Incremental releases of the developed prototype enabled fixes to be monitored and resolved to minimize and mitigate potential risks of a product development [36]. Once the development was completed, the Development Panel approved the final prototype release.

\section{Step 4: Demonstration}

This step focused on the deployment phase of the prototype SDLC. The implementation of the artifact included the deployment of the prototype as developed. The solution was demonstrated to a group of healthcare professionals from varying sectors. An invitation to participate in the demonstration session was sent to each professional. To deploy the prototype, the demonstration incorporated the process of simulation which expanded on two sub-phases of model development: (1) construction of the model and (2) deducing predictions from the developed model [11]. The simulation of the artifact was concerned with all relevant aspects of the model and demonstrated each component within the process from inception to completion. The predictions deduced from the model were typically hypothetical in nature during this phase of the design cycle. To this end, participants of the demonstration phase of the model provided feedback on the core behaviors of the solution: functionality, durability, interoperability impact, data mapping, data translation, and system performance. Discussions with the healthcare professionals were also conducted to determine the usefulness and practical application of the research solution within the respective healthcare sectors. 


\section{Step 5: Evaluation}

One of the objectives of the evaluation phase was the ability to compare the artifact's functionality with its objectives [29]. The validation of the solution was done by the previously selected panel of experts. During the development of the prototype, unit testing was conducted as each developed increment was released. However, an evaluation of the completed prototype was conducted to determine if the research solution met the complete set of requirement specification criteria. Once the prototype of the artifact was developed, the expert panel began a thorough testing process. To achieve this goal, the experts ran a prototype simulation from an online web link. The prototype simulation demonstrated how the artifact was expected to function in a production environment. Artifacts developed in IS research could be evaluated using the process of simulation ([11], [17]). The research artifact was tested for any additional bugs and functional defects. Using a comprehensive test plan the findings of the testing was documented and a complete list was returned to the research facilitator. The test results were consolidated and sent to the panel for final review and approval. At the end of the process, the feedback responses were reviewed by the facilitator and a final test report was completed and provided to the expert reviewers.

\section{Step 6: Communication}

The final results of the research were presented to a group of healthcare professionals with the intent of adding to the body of knowledge in the area of semantic interoperability and design science development. The solution and documentation were also shared with healthcare facilities and participants to provide an endorsement. Documentation of the developed artifact was then prepared based on the design requirements of the approved development.

\section{Summary}

The review of the literature demonstrated that interoperability solutions previously proposed were primarily based on healthcare standards such as openEHR archetypes [7], [25], [26], [47], ISO 13606, semantic ontology using OWL mapping [22], [37], and HL7 standards [22], [39]. RDF, as a standard to achieve interoperability, was not incorporated in any of the proposed solution reviewed in the literature. While these solutions facilitated some interoperability functionality, they were proven to be limited and not scalable enough to allow for the application of new scenarios thus hindering the effective achievement of a broader scope of semantic interoperability [7]. Further, many researchers still claims that semantic interoperability within the healthcare sector has yet to be fully accomplished even with the implementation of the existing systems [9], [16], [22], [37], [47].

Compared to the existing systems, the solution incorporated RDF as its foundation to achieve semantic interoperability. The research model unlike the previous solutions provided a complete package for health systems to achieve true interoperability. The application of RDF to achieve interoperability allowed for multiple data models and vocabularies to be easily combined and interrelated within a single health environment thereby reducing the chances of data ambiguity. Data accuracy and continuity of mappings provided the building blocks of semantic interoperability [37]. These factors were evident in the core of the RDF standard. Accuracy not only referred to the raw data but also included the conformance with federal laws that apply to the achievement of semantic interoperability of healthcare data. Continuity of data mapping referred to the ability to incorporate any changes that occurred in a standard over time, as a result of updates to the standards or federal mandate, and reflecting these changes in the mapping [16]. Overall, using RDF within the translation model ensured that the validity of the data mapped met the level of accuracy necessary for the transformation of different health care standards within that environment thus promoting semantic interoperability.

While the suggested research focused on the development of a single, common information model, further research opportunities and recommendations could include investigations into the implementation of these types of artifacts within a single environment at a multi-facility hospital entity.

\section{References}

[1] Anguita, A., Garcia-Remesal, M., de la Iglesia, D., Graf, N., \& Maojo, V. (2014). Toward a view-oriented approach for aligning RDF-based biomedical repositories. Methods of Information in Medicine, 53(4).

[2] Bahga, A., \& Madisetti, V. K. (2013). A cloud-based approach for interoperable electronic health records (EHRs). IEEE Journal of Biomedical and Health Informatics, 17(5), 894-906.

[3] Botella, F., Alarcon, E., \& Penalver, A. (2014). How to classify to experts in usability evaluation. Interaccion ' 14 : ACM Proceedings of the XV International Conference on Human Computer Interaction. 1-4. 
[4] Bowles, K. H., Potashnik, S., Ratcliffe, S. J., Rosenberg, M., Shih, N.-W., Topaz, M., . . . Naylor, M. D. (2013). Conducting research using the electronic health record across multi-hospital systems: Semantic harmonization implications for administrators. Journal of Nursing Administration, 43(6), 355-360.

[5] Bravo, C., Suarez, C., Gonzalez, C., Lopez, D., \& Blobel, B. (2014). Conceptual model formalization in a semantic interoperability service framework: Transforming relational database schemas to OWL. Studies in Health Technology Informatics, 200, 35-41.

[6] Cimino, J. J. (2007). Collect once, use many: Enabling the reuse of clinical data through controlled terminologies. Journal of AHIMA, 78(2), 24-29.

[7] Costa, C. M., Menárguez-Tortosa, M., \& FernándezBreis, J. T. (2011). Clinical data interoperability based on archetype transformation. Journal of Biomedical Informatics, 44(5), 869-880.

[8] D'Amore, J. D., Mandel, J. C., Kreda, D. A., Swain, A., Koromia, G. A., Sundareswaran, S., . . . Ramoni, R. B. (2014). Are Meaningful Use Stage 2 certified EHRs ready for interoperability? Findings from the SMART C-CDA Collaborative. Journal of the American Medical Informatics Association : JAMIA, 21(6), 1060-1068

[9] Dixon, B. E., Vreeman, D. J., \& Grannis, S. J. (2014). The long road to semantic interoperability in support of public health: Experiences from two states. Journal of biomedical informatics, 49, 3-8.

[10] Duftschmid, G., Rinner, C., Kohler, M., HuebnerBloder, G., Saboor, S., \& Ammenwerth, E. (2013). The EHR-ARCHE project: satisfying clinical information needs in a shared electronic health record system based on IHE XDS and Archetypes. International Journal of Medical Informatics, 82, 1195-1207.

[11] Eekels, J., \& Roozenburg, N.F.M. (1991). A methodological comparison of the structures of scientific research and engineering design: Their similarities and differences. Design Studies, 12(4), 197-203.

[12] EHR/HIE Interoperability Workgroup. (2014). Retrieved September 29, 2014, from http://www.interopwg.org

[13] Fernández-Breis, J. T., Maldonado, J. A., Marcos, M., Legaz-García, M. D. C., Moner, D., Torres-Sospedra, J., . . . Robles, M. (2013). Leveraging electronic healthcare record standards and semantic web technologies for the identification of patient cohorts. Journal of the American Medical Informatics Association, 20(e2), e288-296.

[14] Furukawa, M. F., Patel, V., Charles, D., Swain, M., \& Mostashari, F. (2013). Hospital electronic health information exchange grew substantially in 2008-12. Health affairs (Project Hope), 32(8), 1346-1354.
[15] Gabriel, M. H., Jones, E. B., Samy, L., \& King, J. (2014). Progress and challenges: Implementation and use of health information technology among critical-access hospitals. Health Affairs, 33(7), 1262-1270.

[16] Hammami, R., Bellaaj, H., \& Kacem, A. H. (2014). Interoperability for medical information systems: An overview. Health Technology, 4, 261-272.

[17] Hevner, A. R., March, S. T., Park, J., \& Ram, S. (2004). Design science in information systems research. MIS Quarterly, 28(1), 75-105.

[18] Hosapujari, A. B., \& Verma, A. (2013). Development of a hub and spoke model for bus transit route network design. Procedia - Social and Behavioral Sciences, 104(0), 835-844.

[19] Hsu, C. \& Sandford, B. A. (2007). The Delphi Technique: making sense of consensus. Practical assessment, research \& evaluation, 12(10), 1-7.

[20] Hufnagel S. P. (2009). National electronic health record interoperability chronology. Military Medicine, 5(35), 35-42.

[21] Jones, W., Capra, R., Diekema, A., Teevan, J., PerezQuinones, M., Dinneen, J. D., ... Hemminger, B. (2015). "For Telling" the present: using the Delphi Method to understand personal information management practices. $\mathrm{CHI}$ 15: Proceeding of the $33^{\text {rd }}$ Annual ACM Conference on Human Factors in Computing Systems, 3513-3522.

[22] Khan, W. A., Hussain, M., Afzal, M., Amin, M. B., Saleem, M. A., \& Lee, S. (2013). Personalized-detailed clinical model for data interoperability among clinical standards. Telemedicine Journal and E-health : The official Journal of the American Telemedicine Association, 19(8), 632-642.

[23] Khan, W. A., Khattak, A. M., Hussain, M., Amin, M. B., Afzal, M., Nugent, C., \& Lee, S. (2014). An adaptive semantic based mediation system for data interoperability among Health Information Systems. Journal of Medical Systems, 38(8), 28

[24] Kobayashi, S., Kimura, E., \& Ishihara, K. (2013). Archetype model-driven development framework for EHR web system. Healthcare Informatics Research, 19(4), 271277.

[25] Laleci, G. B., Yuksel, M., \& Dogac, A. (2013). Providing semantic interoperability between clinical care and clinical research domains. IEEE Journal of Biomedical and Health Informatics, 17(2), 356-369.

[26] Menarguez-Tortosa, M. \& Fernandez-Breis, J. T. (2013). OWL-based reasoning methods for validating archetypes. Journal of Biomedical Informatics, 46 (2), 304317. 
[27] Noblin, A., Cortelyou-Ward, K., Cantiello, J., Breyer, T., Oliveira, L., Dangiolo, M., . . . Berman, S. (2013). EHR implementation in a new clinic: a case study of clinician perceptions. Journal of Medical Systems, 37(4), 9955

[28] Ogunyemi, O. I., Meeker, D., Kim, H.-E., Ashish, N., Farzaneh, S., \& Boxwala, A. (2013). Identifying appropriate reference data models for comparative effectiveness research (CER) studies based on data from clinical information systems. Medical Care, 51(8), S45-52.

[29] Peffers, K., Tuunanen, T., Rothenberger, M. A., \& Chatterjee, S. (2007). A design science research methodology for information systems research. Journal of Management Information Systems, 24(3), 45-77

[30] Premkumar, G., Ramamurthy, K., \& Saunders, C. S. (2005). Information processing view of organizations: an exploratory examination of fit in the context of interorganizational relationships. Journal of Management Information Systems, 22(1), 257-294.

[31] Prokosch, H., Ries, M., Beyer, A., Schwenk, M., Seggewies, C., Kopcke, F., ... Burkle, T. (2011). IT infrastructure components to support clinical care and translational research projects in a comprehensive cancer center. Studies in Health Technology and Informatics, 169, 892-896.

[32] Rassafiani, M., Ziviani, J., \& Dalgleish, L. (2009). Identification of occupational therapy clinical exptertise: decision-making characteristics. Australian Occupational Therapy Journal, 56, 156-166.

[33] Saleem, J. J., Flanagan, M. E., Wilck, N. R., Demetriades, J., \& Doebbeling, B. N. (2013). The nextgeneration electronic health record: perspectives of key leaders from the US Department of Veterans Affairs. Journal of the American Medical Informatics Association : JAMIA, 20, e175-e177.

[34] Sao, D., Gupta, A., \& Gantz, D. A. (2013). Interoperable electronic health care record: a case for adoption of a national standard to stem the ongoing health care crisis. Journal of Legal Medicine, 34(1), 55-90

[35] Shanteau, J., Weiss, D. J., Thomas, R. P., \& Pounds, J. C. (2002). Performance-based assessment of expertise: how to decide if someone is an expert or not. European Journal of Operational Research, 136, 253-263.

[36] Shah, U. S. (2016). An excursion to software development life cycle models: an old to ever-growing models. ACM SIGSOFT Software Engineering Notes, 41(1), $1-13$.
[37] Sinaci, A. A., \& Laleci Erturkmen, G. B. (2013). A federated semantic metadata registry framework for enabling interoperability across clinical research and care domains. Journal of Biomedical Informatics, 46(5), 784-794.

[38] Soguero-Ruiz, C., Lechuga-Suárez, L., Mora-Jiménez, I., Ramos-López, J., Barquero-Pérez, Ó., García-Alberola, A., \& Rojo-Álvarez, J. L. (2013). Ontology for heart rate turbulence domain from the conceptual model of SNOMEDCT. IEEE transactions on Bio-Medical Engineering, 60(7), $1825-1833$

[39] Song, T., Hyeoun-Ae, P., \& Jin, D. (2014). Development of health information search engine based on metada and ontology. Healthcare Informatics Research, 20(2), 88-98.

[40] Tao, C., Pathak, J., Solbrig, H. R., Wei, W., \& Chute, C. G. (2011). LexRDF model: an RDF-based unified model for heterogeneous biomedical ontologies. CEUR Workshop Proceedings, 521(3), 1-8.

[41] United States Department of Health and Human Services. (2014). Health information policy. Retrieved September 29, 2014, from

http://www.hhs.gov/ocr/privacy/hipaa/administrative/enforce mentrule/hitechenforcementifr.html.

[42] Vion-Dury, J. (2013). Using RDFs/OWL to ease semantic integration of structured documents. ACM: DocEng '13 Proceedings of the 2013 ACM symposium on Document Engineering, 189-192

[43] Weng, C., Li, Y., Berhe, S., Boland, M. R., Gao, J., Hurby, G. W., ... Bigger, J. T. (2013). An Integrated Model for Patient Care and Clinical Trials (IMPACT) to support clinical research visit scheduling workflow for future learning health systems. Journal of Biomedical Informatics, 46(4), 642-652.

[44] Willighagen, E. L., Waagmeester, A., Spjuth, O., Ansell, P., Williams, A. J., Tkachenko, V., . . . Wild, D. J. (2013). The ChEMBL database as linked open data. Journal of Cheminformatics, 5, 1-12.

[45] World Wide Web Consortium (W3C). Retrieved September 29, 2014, from https://www.w3.org/TR/rdfschema/

[46] Yu, C. H., \& Hunter, J. (2013). Documenting and sharing comparative analyses of 3D digital museum artifacts through semantic web annotations. ACM Journal on Computing and Cultural Heritage, 6(4), 1-20.

[47] Zunner, C., Ganslandt, T., Prokosch, H. U., \& Burkle, T. (2014). A reference architecture for semantic interoperability and its practical application. Studies in Health Technology and Informatics, 198, 40-46. 